\title{
Optimal Product Proliferation in Monopoly: A Dynamic Analysis
}

\author{
LUCA LAMBERTINI \\ Department of Economics, University of Bologna,Italy*
}

\begin{abstract}
The monopolist's incentives towards product proliferation are evaluated in an optimal control model considering three alternative regimes: profit-seeking; social planning; and a hybrid case with monopoly pricing and a regulator setting product innovation to maximise welfare. In equilibtium, the profit-seeking firm supplies a socially suboptimal number of varieties to reduce cannibalisation while the social planner exploits the same effect to satisfy consumers' love for variety and decrease the market price of all products. In terms of the Schumpeter vs Arrow debate on the relationship between market structure and innovation incentives, the results obtained in this model have a definite Arrovian flavour.
\end{abstract}

Keywords: multiproduct firm, product innovation, optimal control

JEL Classifications: L12, O31

\section{Introduction}

Casual observation suggests that firms, more often than not, supply the markets with product ranges rather than single goods. Consumer electronics (PCs, hi-fi components, etc.) and the car industry are two obvious examples, the fashion industry is another. However, the reasons why a firm producing CD players and amplifiers and one producing cars decide to become multiproduct are different. And such incentives also differ from those whereby a fashion firm finds it profitable to produce skirts, jackets together with, e.g., sunglasses and wrist watches. The decision to produce several types of car may be driven by the incentive to exploit economies of scope and scale, as the same engine or chassis can be used for, say, a Golf and an Audi A3. The same argument applies to PCs but not, in general, to hi-fi components, as manufacturing a CD player is essentially different from manufacturing a turntable, a tube amplifier or a loudspeaker, embodying completely different components and technologies. In the latter case, a sound explanation comes from demand-driven aspects as umbrella branding and brand loyalty. In other cases, like the fashion industry, these factors are magnified to such an extent that the firms' product ranges end up including goods that, strictly speaking, do not even belong to the same industry, and are actually outsourced.

*I thank Davide Dragone and Arsen Palestini for precious comments and discussion. The usual disclaimer applies.

(C) 2009 Luca Lambertini. Licenced under the Creative Commons Attribution-Noncommercial 3.0 Licence (http://creativecommons.org/licenses/by-nc/3.0/). Available at http://rofea.org. 


\section{LAMBERTINI Optimal Product Proliferation in Monopoly: A Dynamic Analysis}

There exists a relatively large literature studying the behaviour of multiproduct firms. ${ }^{1}$ In the early studies, the existence of product lines is explained on the grounds of production costs. This is the bottom line of the theory of contestable markets (Baumol, Panzar and Willig, 1982; Panzar, 1989), where the presence of multiproduct firms is justified by economies of scope.

More intriguing, and somewhat less clear-cut, is the role played demand-side factors in determining the incentive for firms to go multiproduct and, if so, to set the optimal product range properly, in view of the negative externality that a new variety exerts on the existing ones whenever they are substitutes (of course this argument does not apply to complements). I.e., any multiproduct firm selling substitute goods has to come to terms with the cannibalisation effect. This aspect must be assessed against the incentive (i) to meet consumers' love for variety on one side and (ii) to fill market niches that could otherwise be exploited by rivals. ${ }^{2}$ This is the essence of the sub-literature analysing product line rivalry in oligopoly, with and without switching costs. In this vein, Brander and Eaton (1984) focus upon the interplay between consumer's demand for differentiated goods on one side, and the strategic and technological effects affecting firms' behaviour, on the other side. Relying on a theoretical model where the analysis confines to Cournot competition, Brander and Eaton verify that firms' strategic decisions as to product range and output level may lead to market equilibria where firms supply product ranges characterised by a high degree of substitutability. This result is derived under the assumption that each firm's product range consists in a given number of varieties, and is therefore subject to a fairly natural critique, namely, that firms may endogenously alter the span of their product range for strategic reasons. This incentive is investigated by Wernerfelt (1986), finding that the driving forces are the heterogeneity of consumer tastes on one side and the cost of product proliferation on the other. The nested logit approach to the same problem reveals that, in a free entry equilibrium, there are too many firms but too few varieties per firm, and the total number of varieties is too small, compared to the social optimum (see Anderson and de Palma, 1992; Anderson, de Palma and Thisse, 1992). The theory of multiproduct firms has evolved along several lines of research, a relevant one taking into account the possibility that consumers bear switching costs (either real or psychological), and consequently consumers' brand loyalty can be so high that they purchase goods from one firm only. As a result, firms' pricing behaviour becomes quasi-collusive (see Klemperer, 1992, 1995; Klemperer an Padilla, 1997 , inter alia). ${ }^{3}$

\footnotetext{
${ }^{1}$ For an exhaustive overview of the theory of multiproduct firms in oligopolistic or perfectly competitive environments, see MacDonald and Slivinsky (1987), Okuguchi and Szidarovsky (1990), De Fraja (1994) and Baranzoni et al. (2003).

${ }^{2} \mathrm{~A}$ more radical possibility is to interpret product proliferation as an instrument to deter entry, or prevent it altogether (see Judd, 1985; Bonanno, 1987).

${ }^{3}$ The behaviour of firms in choosing optimal product lines has also been extensively looked upon in the address approach to product differentiation, under both monopoly and oligopoly (see Mussa and Rosen, 1978; Maskin and Riley, 1984; Gabszewicz et al., 1986; Bonanno, 1987; Champsaur and Rochet, 1989,
} 
Here, I propose a dynamic model using the tools of optimal control theory to study the optimal behaviour of a multiproduct monopolist controlling the vector of prices and the R\&D investment aimed at expanding the number of product varieties over time. An essential ingredient of the model is represented by consumer tastes, characterised by a preference for variety. Three different regimes are considered, alternatively. In the first, the firms is a pure profit-seeking unit. In the second, it is run by a benevolent social planner manoeuvring prices and R\&D for product innovation so as to maximise social welfare. In the third, a hybrid (second best) setting is described, where prices are set so as to maximise profits while the investment to expand the product range is chosen by a regulator that wants to maximise social welfare. I show that a unique saddle point equilibrium exists in each of the three alternative settings. Then, I proceed to compare the optimal R\&D investments, as well as the resulting number of varieties, across regimes. It turns out that the largest number of varieties is supplied in steady state under social planning, while the lowest one is offered by the profit-seeking monopolist, with the product range associated with the hybrid setup located in between. The intuitive reason for this ranking is that expanding the product range is good for consumers as it satisfies their love for variety and, at the same time, drives a decrease in prices due to the negative externality (the so called cannibalisation effect) that each additional variety exerts on existing ones. The cannibalisation problem is precisely the reason why the profit-seeking monopolist refrains from expanding the product range as much as the social planner does. The third case locates in between because the regulator increases the number of varieties in order to soften the pressure exerted by monopoly pricing on consumers. Since the social optimum replicates the perfectly competitive outcome, the ensuing analysis fully fits the terms of the early debate between Schumpeter (1942) and Arrow (1962) focussing on the influence of market structure (or equivalently, market power) on the incentives to invest in innovative activities, yielding a clear-cut Arrovian result.

The remainder of the paper is organised as follows. Section 2 introduces demand and technology. The optimal R\&D portfolio of the monopolist is evaluated in section 3. Section 4 discusses the social optimum, while the second best equilibrium is described in section 5 . The comparative assessment of the three regimes is carried out in section 6. Section 7 contains concluding remarks.

\section{The model}

Consider the following monopoly setting. The market exists over $t \in[0, \infty)$. The firm supplies $n(t) \geq 1$ products, each variety $i=1,2,3 \ldots$ being characterised at any instant $t$ by the following

inter alia). The main issue at stake in this strand of research is the monopolist'ss incentive to distort quality and quantity as compared to the social optimum, and his associated attempt at discriminating among customers with different willingness to pay for quality. 


\section{LAMBERTINI Optimal Product Proliferation in Monopoly: A Dynamic Analysis}

inverse demand function: ${ }^{4}$

$$
p_{i}(t)=\max \left\{0, A-q_{i}(t)-\gamma \sum_{j \neq i} q_{j}(t)\right\} ; \quad A>0,
$$

where the constant parameter $\gamma \in(0,1]$ measures the degree of substitutability between any pair of products. If $\gamma=0$, goods would be independent of each other and the firm would be a monopolist on $n(t)$ independent markets, so that the ensuing analysis would reduce to verifying the profitability of each single product on its own market. Otherwise, if $\gamma=1$, varieties are perfect substitutes, i.e., homogeneous. Usually, in the existing literature of industrial organization, $\gamma$ is considered as being determined by consumers' love for variety, and therefore it is exogenous to the firm. ${ }^{5}$

By investing an amount of resources $k(t)$ at each instant, the monopolist expands the product range according to the following dynamics:

$$
\dot{n}=\alpha k(t)-\beta \gamma, \alpha, \beta>0 .
$$

which establishes that the state variable $n(t)$ is increasing in $k(t)$, with the instantaneous rate of increase being negatively affected by the degree of substitutability $\gamma$. Intuitively, the higher is $\gamma$, the lower must be the incentive for the firm to introduce any additional variety, since this cannibalises profits accruing to the existing ones. The sensitivity of the rate of change in $n(t)$ to the degree of substitutability $\gamma$ is measured by parameter $\beta>0$.

The investment activated to expand the product range involves an instantaneous cost $\Gamma(t)=$ $b[k(t)]^{2}, b>0$, whereby it appears that the innovative activity takes place at decreasing returns to scale. ${ }^{6}$ The production of any given variety requires a constant unit $\operatorname{cost} c \in(0, A)$. Therefore, the instantaneous profit function is:

$$
\pi_{M}(t)=\sum_{i=1}^{n}\left[A-q_{i}(t)-\gamma \sum_{j \neq i} q_{j}(t)-c\right] q_{i}(t)-b k(t)^{2} .
$$

At this point it is worth observing that (3) does not explicitly depend on $n$. Accordingly, in order to build up the optimal control problem of the firm, one has first to determine, in a quasi-static

\footnotetext{
${ }^{4}$ This demand structure was introduced by Bowley (1924), and it has been used, more recently, by several authors (see Spence, 1976; Dixit, 1979; Singh and Vives, 1984; inter alia).

${ }^{5}$ An interesting extension of the present model would consist in allowing the firm to invest in R\&D to modify $\gamma$. The analysis of this problem in a differential oligopoly game among single-product firms is in Cellini and Lambertini $(2002,2004)$. Another possible extension consists in studying process and product innovation simultaneously (see Lambertini, 2003, and the references therein).

${ }^{6}$ This specific aspect of the R\&D technology will play a crucial role in determining the relative incentives to expand the product range under monopoly, social planning and the second best setting. See section 6 .
} 
way, the optimal output chosen by the monopolist for each variety $i$ at a generic time $t \in[0, \infty){ }^{7}$ The first order condition (FOC) for profit maximisation w.r.t. variety $i$ is the following:

$$
\frac{\partial \pi_{M}(t)}{\partial q_{i}}=A-2\left(q_{i}(t)+\gamma \sum_{j \neq i} q_{j}(t)\right)-c=0 .
$$

Introducing the symmetry condition $q_{i}(t)=q_{j}(t)=q(t)$, the above FOC can be rewritten as follows:

$$
\frac{\partial \pi_{M}(t)}{\partial q_{i}}=A-2[1+\gamma(n(t)-1)] q(t)-c=0,
$$

yielding

$$
q_{M}^{*}(t)=\frac{A-c}{2[1+\gamma(n(t)-1)]}
$$

as the optimal output level per-variety. The overall monopoly output in equilibrium is then $Q_{M}^{*}(t)=n(t)(A-c) /\{2[1+\gamma(n(t)-1)]\}$. The non-negativity of both per-variety and overall output entails the following constraint on $n(t)$ and $\gamma$ :

$$
q_{M}^{*}(t), Q_{M}^{*}(t)>0 \text { iff } \gamma>-\frac{1}{n(t)-1}
$$

which is met for all $\gamma \in(0,1]$. The equilibrium price is $p_{M}^{*}=(A-c) / 2$ for each variety. Equilibrium profits, given the marginal cost and the product line, are:

$$
\pi_{M}^{*}(t)=\frac{n(t)(A-c)^{2}}{4[1+\gamma(n(t)-1)]}-b[k(t)]^{2} .
$$

Again, condition (7) must hold in order for $n(t)(A-c)^{2} / 4[1+\gamma(n(t)-1)]>0$, i.e., the operative profits (gross of all innovation costs) must be positive.

The firm must choose $k(t)$ so as to maximise the discounted profit flow:

$$
\Pi_{M} \equiv \int_{0}^{\infty} \pi_{M}^{*}(t) e^{-\rho t} d t, \rho>0
$$

under the constraint (2). The dynamic problem of the profit-maximising monopolist is investigated in the next section.

\section{The optimal product range under monopoly}

The profit-seeking monopoly equilibrium is summarised in the following:

Proposition 1 Assume $(A-c)^{2} \geq[2+4 b \beta(1-\gamma) \gamma \rho]^{2} /\left[2 b \alpha^{2} \beta(1-\gamma) \gamma \rho\right]$. If so, then the pair

$$
k_{M}^{*}=\frac{\beta \gamma}{\alpha} ; n_{M}^{*}=-\frac{1-\gamma}{\gamma}+\frac{\alpha(A-c) \sqrt{2 b \beta \gamma(1-\gamma) \rho}}{4 b \beta \gamma^{2} \rho} \geq 2
$$

is the unique saddle point equilibrium for the multiproduct monopolist.

${ }^{7}$ Being this a monopoly market, one could equivalently take prices as the relevant varibles. Indeed, in the remainder I will often refer to "monopoly pricing". 
LAMBERTINI Optimal Product Proliferation in Monopoly: A Dynamic Analysis

Proof. The firm's Hamiltonian is:

$$
\mathcal{H}_{M}=e^{-\rho t}\left\{\frac{n(t)(A-c)^{2}}{4[1+\gamma(n(t)-1)]}-b[k(t)]^{2}+\lambda(t)[\alpha k(t)-\beta \gamma]\right\}
$$

where $\lambda(t)=\mu(t) e^{\rho t}$ is the co-state variable (evaluated at time $t$ ) associated with the state variable $n(t)$. The initial condition is $n(0) \geq 1$, and the first order condition on the investment is: ${ }^{8}$

$$
\frac{\partial \mathcal{H}_{M}}{\partial k}=-2 b k+\alpha \lambda=0
$$

yielding:

$$
\lambda=\frac{2 b k}{\alpha} ; \dot{k}=\frac{\alpha \dot{\lambda}}{2 b}
$$

where $\lambda$ is given by the co-state equation:

$$
-\frac{\partial \mathcal{H}_{M}}{\partial n}=\dot{\lambda}-\rho \lambda \Leftrightarrow \dot{\lambda}=\rho \lambda-\frac{(A-c)^{2}(1-\gamma)}{4[1+\gamma(n-1)]^{2}} .
$$

The transversality condition is $\lim _{t \rightarrow \infty} \mu n=0 .{ }^{9}$ Using (12-13), the dynamics of $k$ can be written as follows:

$$
\dot{k}=\frac{\alpha}{2 b}\left(\frac{2 b \rho k}{\alpha}-\frac{(A-c)^{2}(1-\gamma)}{4[1+\gamma(n-1)]^{2}}\right)
$$

and imposing $\dot{k}=0$ yields the optimal investment in terms of the product range and the set of parameters:

$$
k_{M}^{*}(n)=\frac{\alpha(A-c)^{2}(1-\gamma)}{8 b \rho[1+\gamma(n-1)]^{2}}
$$

which can be substituted into (2). Finally, $\dot{n}=0$ has two roots:

$$
n=-\frac{1-\gamma}{\gamma} \pm \frac{\alpha(A-c) \sqrt{2 b \beta \gamma(1-\gamma) \rho}}{4 b \beta \gamma^{2} \rho}
$$

with the smaller root being negative for all admissible values of parameters $\{A, b, c, \alpha, \beta, \gamma, \rho\}$. Therefore, the larger root candidates as the unique economically relevant solution. The monopolist produces at least two varieties if ${ }^{10}$

$$
(A-c)^{2} \geq \frac{[2+4 b \beta(1-\gamma) \gamma \rho]^{2}}{2 b \alpha^{2} \beta(1-\gamma) \gamma \rho} .
$$

\footnotetext{
${ }^{8}$ In the remainder, both the time argument and exponential discounting are omitted for the sake of brevity. ${ }^{9}$ By applying Arrow's sufficiency condition (Arrow, 1968), it is easily shown that the problem is concave over the whole admissible range of parameters.

${ }^{10}$ One has to check the condition ensuring $n \geq 2$ since, if it were $n=1$, then the problem would be trivial as the firm would not supply more than a single variety.
} 
Condition (17) says, intuitively, that expanding the product range is profitable provided that the market be "large enough". If the above inequality is met, then in correspondence of

$$
n_{M}^{*}=-\frac{1-\gamma}{\gamma}+\frac{\alpha(A-c) \sqrt{2 b \beta \gamma(1-\gamma) \rho}}{4 b \beta \gamma^{2} \rho},
$$

the steady state investment is $k_{M}^{*}=\beta \gamma / \alpha$.

The stability analysis can be carried out by studying the Jacobian matrix based upon differential equations (2) and (14):

$$
J=\left[\begin{array}{cc}
\frac{\partial \dot{n}}{\partial n}=0 & \frac{\partial \dot{n}}{\partial \dot{k}}=\alpha \\
\frac{\partial \dot{k}}{\partial n}=\frac{\alpha(A-c)^{2}(1-\gamma) \gamma}{4 b[1+\gamma(n-1)]^{3}} & \frac{\partial \dot{k}}{\partial k}=\rho
\end{array}\right]
$$

whose trace and determinant are:

$$
\begin{gathered}
\operatorname{Tr}(J)=\rho \\
\Delta(J)=-\frac{\alpha^{2}(A-c)^{2}(1-\gamma) \gamma}{4 b[1+\gamma(n-1)]^{3}} .
\end{gathered}
$$

Since $\Delta(J)<0$ everywhere, the steady state is a saddle point

Now we can examine the properties of the steady state product range in terms of comparative statics on parameters:

Corollary 1 In the admissible parameter range, the following properties hold:

$$
\frac{\partial n_{M}^{*}}{\partial \alpha}>0 ; \frac{\partial n_{M}^{*}}{\partial b}<0 ; \frac{\partial n_{M}^{*}}{\partial \beta}<0 ; \frac{\partial n_{M}^{*}}{\partial \gamma}<0 ; \frac{\partial n_{M}^{*}}{\partial \rho}<0 .
$$

Proof. It follows trivially from examining the partial derivatives:

$$
\begin{aligned}
\frac{\partial n_{M}^{*}}{\partial \alpha} & =\frac{(A-c) \sqrt{2 b \beta \gamma(1-\gamma) \rho}}{4 b \beta \gamma^{2} \rho} ; \frac{\partial n_{M}^{*}}{\partial b}=-\frac{(A-c) \alpha \beta(1-\gamma)^{2} \rho}{4 \sqrt{2[b \beta \gamma(1-\gamma) \rho]^{3}}} \\
\frac{\partial n_{M}^{*}}{\partial \beta} & =-\frac{(A-c) b \alpha(1-\gamma)^{2} \rho}{4 \sqrt{2[b \beta \gamma(1-\gamma) \rho]^{3}}} ; \frac{\partial n_{M}^{*}}{\partial \rho}=-\frac{(A-c) b \alpha \beta(1-\gamma)^{2}}{4 \sqrt{2[b \beta \gamma(1-\gamma) \rho]^{3}}}
\end{aligned}
$$

Then, observe that:

$$
\frac{\partial n_{M}^{*}}{\partial \gamma}=\frac{8 \sqrt{b \beta \gamma(1-\gamma) \rho}-(A-c) \alpha(3-2 \gamma) \sqrt{2}}{8 \gamma^{2} \sqrt{b \beta \gamma(1-\gamma) \rho}}<0
$$

for all

$$
(A-c)^{2}>\frac{32 b \beta \gamma(1-\gamma) \rho}{\alpha^{2}(3-2 \gamma)^{2}}
$$




\section{LAMBERTINI Optimal Product Proliferation in Monopoly: A Dynamic Analysis}

However, since

$$
\frac{32 b \beta \gamma(1-\gamma) \rho}{\alpha^{2}(3-2 \gamma)^{2}}<\frac{[2+4 b \beta(1-\gamma) \gamma \rho]^{2}}{2 b \alpha^{2} \beta(1-\gamma) \gamma \rho}
$$

always, then $\partial n_{M}^{*} / \partial \gamma<0$

The interpretation of the above Corollary is straightforward. The optimal product range increases monotonically in the efficiency of $\mathrm{R} \& \mathrm{D}$ efforts (measured by parameter $\alpha$ ), while decreases in its marginal cost $(b)$; in the degree of product substitutability $(\gamma)$ and its weight $(\beta)$ in the kinematic equation (2); as well as in discounting $(\rho)$. In particular, two features are worth stressing: (i) the negative effect exerted by product substitutability on the extent of the product range is due to the fact that, as products become more similar (or, as the consumer taste for variety shrinks), expanding the range magnifies the cannibalisation effect of any additional variety on the existing ones; and (ii) the higher the discount rate, the lower are perceived future returns from any additional variety introduced by the firm; therefore, the product range is negatively affected by discounting.

\section{Social optimum}

As in the previous case, I first solve the quasi-static problem concerning the output (or price) for any single variety, given the technology and the product range. At any instant, the planner maximises social welfare, defined as the sum of profits and consumer surplus:

$$
\begin{aligned}
s w & =\pi+c s=\sum_{i=1}^{n}\left[A-q_{i}-\gamma \sum_{j \neq i} q_{j}-c\right] q_{i}+ \\
& +\sum_{i=1}^{n} \frac{\left(A-p_{i}\right) q_{i}}{2}-b k(t)^{2}
\end{aligned}
$$

w.r.t. the vector $\mathbf{Q} \equiv\left\{q_{1}, \ldots q_{i}, \ldots q_{n}\right\}$. The FOC w.r.t. the output level of variety $i$ is:

$$
\frac{\partial s w}{\partial q_{i}}=A-q_{i}[1+\gamma(n-1)]-c=0
$$

yielding, for a given product range, the optimal output:

$$
q_{S P}^{*}=\frac{A-c}{1+\gamma(n(t)-1)}
$$

which can be compared to (6) to verify immediately that $q_{S P}^{*}=2 q_{M}^{*}$ for all $n$. Obviously $Q_{S P}^{*}=$ $2 Q_{M}^{*}$, while $p_{S P}^{*}=c$. Therefore,

$$
s w_{S P}^{*}(t)=c s_{S P}^{*}(t)=\frac{n(t)(A-c)^{2}}{2[1+\gamma(n(t)-1)]}-b[k(t)]^{2} .
$$


The firm must choose $k(t)$ so as to maximise the discounted flow of social welfare:

$$
S W_{S P} \equiv \int_{0}^{\infty} s w_{S P}^{*}(t) e^{-\rho t} d t, \rho>0
$$

under the constraint (2). The dynamic problem of the welfare-maximising planner is investigated in the next subsection. Before proceeding with the details of calculations, it is worth noting the analogy between the social optimum and the performance of a perfectly competitive industry where $n(t)$ single-product firms enter the market over time. The fact that the cost function $\Gamma(t)$ remains a single one can be justified by thinking of a common pool of technological knowledge available to all firms alike under perfect competition. ${ }^{11}$ This analogy will play a relevant role in assessing the long run performance of the industry in terms of innovation incentives across regimes.

\subsection{The optimal product range under social planning}

The social planning (or first best) equilibrium is summarised in the following:

Proposition 2 Assume $(A-c)^{2} \geq 4 b \beta(1+\gamma)^{2} \gamma \rho /\left[\alpha^{2}(1-\gamma)\right]$. If so, then the pair

$$
k_{S P}^{*}=\frac{\beta \gamma}{\alpha} ; n_{S P}^{*}=-\frac{1-\gamma}{\gamma}+\frac{\alpha(A-c) \sqrt{b \beta \gamma(1-\gamma) \rho}}{2 b \beta \gamma^{2} \rho} \geq 2
$$

is the unique saddle point equilibrium for the social planner.

Proof. The benevolent planner's Hamiltonian is:

$$
\mathcal{H}_{S P}=e^{-\rho t}\left\{\frac{n[\alpha-c(k)]^{2}}{2[1+\gamma(n-1)]}-b[k(t)]^{2}+\lambda(t)[\alpha k(t)-\beta \gamma]\right\}
$$

where $\lambda(t)=\mu(t) e^{\rho t}$ is the co-state variable (evaluated at time $t$ ) associated with the state variable $n(t)$. The initial condition is $n(0) \geq 1$, and the first order condition on the investment is:

$$
\frac{\partial \mathcal{H}_{S P}}{\partial k}=-2 b k+\alpha \lambda=0
$$

yielding:

$$
\lambda=\frac{2 b k}{\alpha} ; \dot{k}=\frac{\alpha \dot{\lambda}}{2 b}
$$

where $\lambda$ is given by the co-state equation:

$$
-\frac{\partial \mathcal{H}_{S P}}{\partial n}=\dot{\lambda}-\rho \lambda \Leftrightarrow \dot{\lambda}=\rho \lambda-\frac{(A-c)^{2}(1-\gamma)}{2[1+\gamma(n-1)]^{2}} .
$$

\footnotetext{
${ }^{11}$ This is the common view in the literature on innovation and growth under monopolistic competition (see Grossman and Helpman, 1991, inter alia). Obviously, such an assumption would be hardly justified under oligopoly.
} 


\section{LAMBERTINI Optimal Product Proliferation in Monopoly: A Dynamic Analysis}

The transversality condition is $\lim _{t \rightarrow \infty} \mu n=0$. Using (33-34), the dynamics of $k$ can be written as follows:

$$
\dot{k}=\frac{\alpha}{2 b}\left(\frac{2 b \rho k}{\alpha}-\frac{(A-c)^{2}(1-\gamma)}{2[1+\gamma(n-1)]^{2}}\right)
$$

and imposing $k=0$ yields the optimal investment in terms of the product range and the set of parameters:

$$
k_{S P}^{*}(n)=\frac{\alpha(A-c)^{2}(1-\gamma)}{4 b \rho[1+\gamma(n-1)]^{2}}
$$

which can be substituted into (2). Finally, $\dot{n}=0$ has two roots:

$$
n=-\frac{1-\gamma}{\gamma} \pm \frac{\alpha(A-c) \sqrt{b \beta \gamma(1-\gamma) \rho}}{2 b \beta \gamma^{2} \rho}
$$

with the smaller root being negative for all admissible values of parameters $\{A, b, c, \alpha, \beta, \gamma, \rho\}$. Therefore, the larger root candidates as the economically relevant solution. The monopolist produces at least two varieties if

$$
(A-c)^{2} \geq \frac{4 b \beta(1+\gamma)^{2} \gamma \rho}{\alpha^{2}(1-\gamma)}
$$

In correspondence of

$$
n_{S P}^{*}=-\frac{1-\gamma}{\gamma}+\frac{\alpha(A-c) \sqrt{b \beta \gamma(1-\gamma) \rho}}{2 b \beta \gamma^{2} \rho},
$$

the steady state investment is $k_{S P}^{*}=\beta \gamma / \alpha$.

In this setting, the relevant Jacobian matrix is based upon differential equations (2) and (35):

$$
J=\left[\begin{array}{cc}
\frac{\partial \dot{n}}{\partial n}=0 & \frac{\partial \dot{n}}{\partial \dot{k}}=\alpha \\
\frac{\partial \dot{k}}{\partial n}=\frac{\alpha(A-c)^{2}(1-\gamma) \gamma}{2 b[1+\gamma(n-1)]^{3}} & \frac{\partial \dot{k}}{\partial k}=\rho
\end{array}\right]
$$

The trace and determinant of matrix $J$ are:

$$
\begin{gathered}
\operatorname{Tr}(J)=\rho \\
\Delta(J)=-\frac{\alpha^{2}(A-c)^{2}(1-\gamma) \gamma}{2 b[1+\gamma(n-1)]^{3}} .
\end{gathered}
$$

Since $\Delta(J)<0$ everywhere, the steady state is a saddle point

The equivalent of Corollary 1 can be shown to apply also in the social planning case: 
Corollary 2 In the admissible parameter range, the following properties hold:

$$
\frac{\partial n_{S P}^{*}}{\partial \alpha}>0 ; \frac{\partial n_{S P}^{*}}{\partial b}<0 ; \frac{\partial n_{S P}^{*}}{\partial \beta}<0 ; \frac{\partial n_{S P}^{*}}{\partial \gamma}<0 ; \frac{\partial n_{S P}^{*}}{\partial \rho}<0 .
$$

Proof. Omitted for brevity, as it follows the same lines as for Corollary 1

From Propositions 1-2, we immediately draw the following implication:

Corollary $3 k_{S P}^{*}=k_{M}^{*}$, while $n_{S P}^{*}-n_{M}^{*} \propto(2-\sqrt{2})(A-c) \alpha(1-\gamma)>0$ for all $\gamma \in(0,1)$.

The fact that the steady state $R \& D$ investment is the same in the two regimes is a direct consequence of the state dynamics. Instead, the suboptimality of the optimal monopoly product range is clearly driven by profit incentives. A planner that aims at maximising welfare will end up offering more varieties just because by doing so the resulting industry output (price) increases (decreases), enhancing consumer surplus.

However, this may not be the most appropriate comparison, because it relies on the assumption that the planner controls not only investment but also output levels. A more realistic approach is a second best perspective where a regulator controls R\&D efforts, given the optimal output (or price) chosen by the profit-seeking monopolist for each existing variety.

\section{Second best}

The optimal monopoly output for each variety, taking the number of varieties as given, is (6). Therefore, the instantaneous social welfare in second best is:

$$
s w_{S B}^{*}(t)=\pi_{S B}^{*}(t)+c s_{S B}^{*}(t)
$$

where $\pi_{S B}^{*}(t)=\pi_{M}^{*}(t)$ as in (8) and consumer surplus is:

$$
c s_{S B}^{*}(t)=\frac{n(t)(A-c)^{2}}{8[1+\gamma(n(t)-1)]} .
$$

Accordingly, the regulator chooses $k(t)$ so as to maximise:

$$
S W_{S B} \equiv \int_{0}^{\infty} s w_{S B}^{*}(t) e^{-\rho t} d t, \rho>0
$$

under the constraint (2). The dynamic problem of the welfare-maximising regulator is investigated in the next subsection.

\subsection{The second best product range}

The second best equilibrium is summarised in the following: 
LAMBERTINI Optimal Product Proliferation in Monopoly: A Dynamic Analysis

Proposition 3 Assume $(A-c)^{2} \geq 16 b \beta(1+\gamma)^{2} \rho /\left[3 \alpha^{2} \gamma(1-\gamma)\right]$. If so, then the pair

$$
k_{S B}^{*}=\frac{\beta \gamma}{\alpha} ; n_{S B}^{*}=-\frac{1-\gamma}{\gamma}+\frac{\alpha(A-c) \sqrt{3 b \beta \gamma(1-\gamma) \rho}}{4 b \beta \gamma^{2} \rho} \geq 2
$$

is the unique saddle point equilibrium for the regulator.

Proof. The regulator's Hamiltonian is:

$$
\mathcal{H}_{S B}=e^{-\rho t}\left\{\frac{3 n(A-c)^{2}}{8[1+\gamma(n-1)]}-b[k(t)]^{2}+\lambda(t)[\alpha k(t)-\beta \gamma]\right\}
$$

where $\lambda(t)=\mu(t) e^{\rho t}$ is the co-state variable (evaluated at time $t$ ) associated with the state variable $n(t)$. The initial condition is $n(0) \geq 1$, and the first order condition on the investment is:

$$
\frac{\partial \mathcal{H}_{S B}}{\partial k}=-2 b k+\alpha \lambda=0
$$

yielding:

$$
\lambda=\frac{2 b k}{\alpha} ; \dot{k}=\frac{\alpha \dot{\lambda}}{2 b}
$$

where $\lambda$ is given by the co-state equation:

$$
-\frac{\partial \mathcal{H}_{S B}}{\partial n}=\dot{\lambda}-\rho \lambda \Leftrightarrow \dot{\lambda}=\rho \lambda-\frac{3(A-c)^{2}(1-\gamma)}{8[1+\gamma(n-1)]^{2}} .
$$

The transversality condition is $\lim _{t \rightarrow \infty} \mu n=0$. Using (48-49), the dynamics of $k$ can be written as follows:

$$
\dot{k}=\frac{\alpha}{2 b}\left(\frac{2 b \rho k}{\alpha}-\frac{3(A-c)^{2}(1-\gamma)}{8[1+\gamma(n-1)]^{2}}\right)
$$

and imposing $\dot{k}=0$ yields the optimal investment in terms of the product range and the set of parameters:

$$
k_{S B}^{*}(n)=\frac{3 \alpha(A-c)^{2}(1-\gamma)}{16 b \rho[1+\gamma(n-1)]^{2}}
$$

which can be substituted into (2). Finally, $\dot{n}=0$ has two roots:

$$
n=-\frac{1-\gamma}{\gamma} \pm \frac{\alpha(A-c) \sqrt{3 b \beta \gamma(1-\gamma) \rho}}{4 b \beta \gamma^{2} \rho}
$$

with the smaller root being negative for all admissible values of parameters $\{A, b, c, \alpha, \beta, \gamma, \rho\}$. Therefore, the larger root candidates as the economically relevant solution. The firm produces at least two varieties if

$$
(A-c)^{2} \geq \frac{16 b \beta(1+\gamma)^{2} \rho}{3 \alpha^{2} \gamma(1-\gamma)}
$$


In correspondence of

$$
n_{S B}^{*}=-\frac{1-\gamma}{\gamma}+\frac{\alpha(A-c) \sqrt{3 b \beta \gamma(1-\gamma) \rho}}{4 b \beta \gamma^{2} \rho},
$$

the steady state investment is $k_{S B}^{*}=\beta \gamma / \alpha$.

The stability properties of the dynamic system of differential equations (2) and (35) must be assessed using the Jacobian matrix:

$$
J=\left[\begin{array}{cc}
\frac{\partial \dot{n}}{\partial n}=0 & \frac{\partial \dot{n}}{\partial k}=\alpha \\
\frac{\partial \dot{k}}{\partial n}=\frac{3 \alpha(A-c)^{2}(1-\gamma) \gamma}{8 b[1+\gamma(n-1)]^{3}} & \frac{\partial \dot{k}}{\partial k}=\rho
\end{array}\right]
$$

whose trace and determinant are:

$$
\begin{gathered}
\operatorname{Tr}(J)=\rho \\
\Delta(J)=-\frac{3 \alpha^{2}(A-c)^{2}(1-\gamma) \gamma}{8 b[1+\gamma(n-1)]^{3}} .
\end{gathered}
$$

As $\Delta(J)<0$ over the whole admissible range of parameters, it appears that the steady state is a saddle point.

The properties $\partial n_{S B}^{*} / \partial \alpha>0 ; \partial n_{S B}^{*} / \partial b<0 ; \partial n_{S B}^{*} / \partial \beta<0 ; \partial n_{S B}^{*} / \partial \gamma<0$ and $\partial n_{S B}^{*} / \partial \rho<0$ are easily proved.

\section{Comparing R\&D incentives across regimes}

Now I am in a position to comparatively assess the incentives to expand the product range across the three different regimes examined so far. This is easily done by evaluating expressions (15), (36) and (51) corresponding to $k_{M}^{*}(n), k_{S P}^{*}(n)$ and $k_{S B}^{*}(n)$, yielding

Proposition $4 k_{S P}^{*}(n)>k_{S B}^{*}(n)>k_{M}^{*}(n)$ over the whole admissible range of parameters $\{A, b, c, \alpha, \beta, \gamma, \rho\}$.

The fact that $k_{S P}^{*}(n)>k_{M}^{*}(n)$ is indeed intuitive. The associated result whereby $k_{S B}^{*}(n) \in$ $\left(k_{M}^{*}(n), k_{S P}^{*}(n)\right)$ can be explained by recalling that the second best product range $k_{S B}^{*}(n)$ is attained in the hybrid case where the pricing rule is determined by the profit-seeking monopolist while the $\mathrm{R} \& \mathrm{D}$ activity for product innovation is controlled by a welfare-maximising regulator, who, in a sense, has a taste for cannibalisation (which is precisely what the firm would like to avoid as much as possible). This preference for cannibalisation clearly arises because expanding the product range softens the harmful effects of monopoly pricing on consumer surplus. An obvious consequence of Proposition 4 is 


\section{LAMBERTINI Optimal Product Proliferation in Monopoly: A Dynamic Analysis}

Corollary $4 n_{S P}^{*}>n_{S B}^{*}>n_{M}^{*}$ over the whole admissible range of parameters $\{A, b, c, \alpha, \beta, \gamma, \rho\}$.

As a last step, it is also easily checked that $s w\left(n_{S P}^{*}, k_{S P}^{*}\right)>s w\left(n_{S B}^{*}, k_{S B}^{*}\right)>s w\left(n_{M}^{*}, k_{M}^{*}\right)$, which, amongst other things, confirms that in the second best equilibrium the increase in consumer surplus outweighs the increase in R\&D costs, i.e., cannibalisation is an instrument in the hands of the regulator to increase social welfare in situations where he cannot directly control the pricing rule.

Making one step back from the steady state equilibrium to the control dynamics, one can evaluate the trajectories of controls and states towards the long run equilibrium. Suppose we want to compare the rates of change of optimal investments in the three regimes. This exercise can be carried out on the basis of the control equations (14), (35) and (50), under the useful and plausible assumption that the initial condition on the state variable, $n(0)=n_{0}$, be the same across regimes. Then, at $t=0$, one obtains:

$$
\dot{k}_{M}>\dot{k}_{S B}>\dot{k}_{S P}
$$

for any admissible values of parameters $\{A, b, c, \alpha, \beta, \gamma, \rho\}$. That is, at the initial instant, the trajectory of the monopolist's optimal R\&D effort is steeper than the remaining two; conversely, that of the social planner's control is flatter than the other two. However, from Proposition 4 and Corollary 4 we know that, in equilibrium, monopoly (respectively, social planning) is characterised by the lowest (resp., highest) investment. This finding has a clear-cut implications and an intuitive explanation: the implication is that the trajectories characterising the three regimes intersect at some intermediate date, while the explanation is that the profit-seeking monopolist finds it convenient to approach the long run optimal investment effort as fast as possible while exactly the opposite holds for the welfare-maximising planner who wants to smooth the overall $R \& D$ cost over the infinitely long time horizon available. That is, the planner takes longer than a profit-seeking firm to supply any given number of goods because going slow helps reducing the overall cost of development borne by society. ${ }^{12}$ The (hybrid) second best case lies necessarily in between. A qualitative description of this aspect of the model is in Figure 1 , where I assume, without further loss of generality, $k(0)=0$ in all regimes, and examine the relative speed at which the firm approaches $k_{i}^{*}(n)$, for a given $n .^{13}$

The picture clearly shows that the profit-seeking monopoly underinvests in product innovation/proliferation as compared to the other two regimes. The immediate implication of this is that the foregoing analysis prompts for the introduction of some form or regulation of innovative activity (as in the hybrid regime $S B$ ), and/or for the adoption of R\&D subsidies (which could

\footnotetext{
${ }^{12}$ In a nutshell, the attitude of the social planner in this respect is summarised by a Jensen's inequality applied to the convex instantaneous R\&D cost function $\Gamma(t)=b[k(t)]^{2}$.

${ }^{13}$ Using (14), (35) and (50) one can perform numerical simulations reflecting the picture represented in Figures 1.
} 
be easily represented here by a decrease of parameter $b$, or an increase in parameter $\alpha$ ) in order to counterbalance or eliminate altogether the socially undesirable effects of cannibalisation on the monopolist's willingness to expand the product range.

Figure 1: The ranking of R\&D efforts over time, given $n$

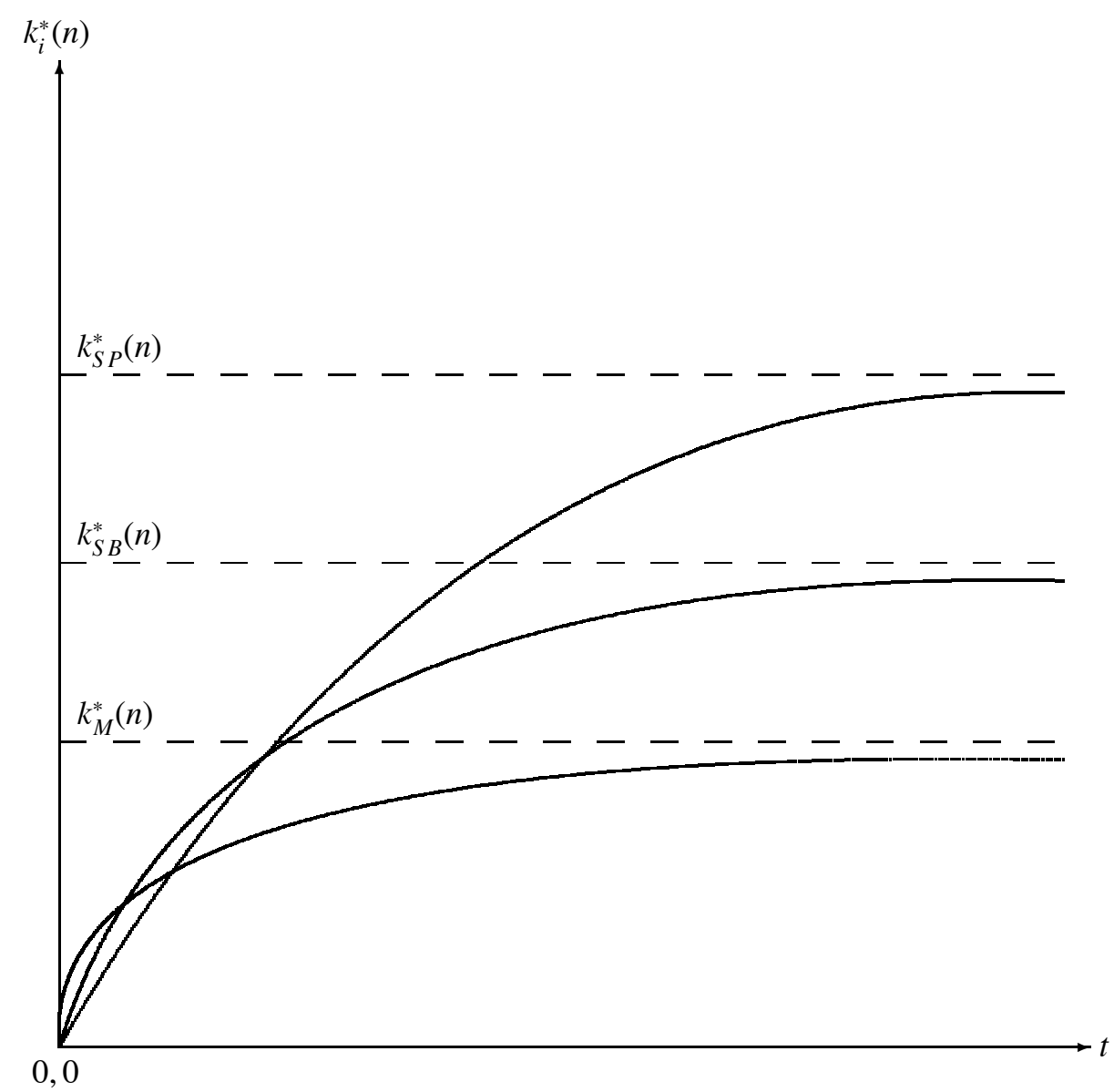

An overall appraisal of the comparative evaluation of $R \& D$ efforts and the resulting optimal product ranges characterising monopoly on one side and social planning on the other can be made by nesting the present model into the original terms of the debate between Schumpeter (1942) and Arrow (1962) about the bearings of market structure on R\&D activities. Here, social planning (or perfect competition) definitely outperforms monopoly, so that the flavour of this model appears to be clearly Arrovian. 
LAMBERTINI Optimal Product Proliferation in Monopoly: A Dynamic Analysis

\section{Concluding remarks}

I have taken a dynamic approach to the analysis of the optimal investment in R\&D activities aimed at enlarging the spectrum of varieties offered by a multiproduct monopolist under three alternative perspectives: one where the firm is a pure profit-seeking unit; another where it is run by a benevolent planner; and the last where prices are set by profit-seeking shareholders while a public agency regulates the $R \& D$ activity for product innovation. The main message emerging from the foregoing investigation is that cannibalisation indeed plays a major role in shaping the optimal size of the spectrum of varieties. In particular, smoothing the $R \& D$ costs over time, the social planner outperforms the profit-seeking monopolist along this specific dimension, as any additional good introduced in the market has a twofold beneficial effect: it meets consumers' preference for variety and brings about an increase in industry output as well as a decrease in the market price of all products alike. Both effects operate an increase in consumer surplus and ultimately also in social welfare. In view of the equivalence between social planning and perfect competition, this outcome can be considered as a well defined Arrovian result.

\section{Reference}

Anderson, S. and A. de Palma (1992), Multiproduct Firms: A Nested Logit Approach, Journal of Industrial Economics, 40, 261-76.

Anderson, S., A. de Palma and J.-F. Thisse (1992), Discrete Choice Theory of Product Differentiation, Cambridge, MA, MIT Press.

Arrow, K.J. (1962), Economic Welfare and the Allocation of Resources for Invention, in R. Nelson (ed.), The Rate and Direction of Industrial Activity, Princeton, NJ, Princeton University Press.

Arrow, K.J. (1968), Applications of Control Theory to Economic Growth, in G.B. Dantzig and A.F. Veinott, Jr (eds), Mathematics of the Decision Sciences, Part 2, American Mathematical Society, Providence.

Baranzoni, S., P. Bianchi and L. Lambertini (2003), Multiproduct Firms, in P. Bianchi and L. Lambertini (eds), Technology, Information and Market Dynamics: Topics in Advanced Industrial Organization, Cheltenham, Edward Elgar, 51-80.

Baumol, W., J. Panzar and R. Willig (1982), Contestable Markets and the Theory of Industry Structure, New York, Harcourt Brace Jovanovich.

Bonanno, G. (1987), Location Choice, Product Proliferation and Entry Deterrence, Review of Economic Studies, 54, 37-46.

Bowley, A.L. (1924), The Mathematical Groundwork of Economics, Oxford, Oxford University Press.

Brander, J. and J. Eaton (1984), Product Line Rivalry, American Economic Review, 74, 323-34. 
Cellini, R., and L. Lambertini (2002), A Differential Game Approach to Investment in Product Differentiation, Journal of Economic Dynamics and Control, 27, 51-62.

Cellini, R. and L. Lambertini (2004), Private and Social Incentives Towards Investment in Product Differentiation, International Game Theory Review, 6, 493-508.

Champsaur, P. and J.-C. Rochet (1989), Multiproduct Duopolists, Econometrica, 57, 533-57.

De Fraja, G. (1994), A General Characterization of Multiproduct Cournot Competition, Bulletin of Economic Research, 46, 171-83.

Dixit, A. (1979), A Model of Duopoly Suggesting a Theory of Entry Barriers, Bell Journal of Economics, 10, 20-32.

Gabszewicz, J.J., A. Shaked, J. Sutton and J.-F. Thisse (1986), Segmenting the Market: The Monopolist Optimal Product Mix, Journal of Economic Theory, 39, 273-89.

Grossman, G. and E. Helpman (1991), Innovation and Growth in the Global Economy, Cambridge, MA, MIT Press.

Judd, K. (1985), Credible Spatial Preemptition, RAND Journal of Economics, 16, 153-66.

Klemperer, P. (1992), Equilibrium Product Lines: Competing Head-to-Head May Be Less Competitive, American Economic Review, 82, 740-55.

Klemperer, P. (1995), Competition when Consumers Have Switching Costs: An Overview with Applications to Industrial Organization, Macroeconomics, and International Trade, Review of Economic Studies, 62, 515-39.

Klemperer, P. and A.J. Padilla (1997), Do Firms' Product Lines Include Too Many Varieties?, RAND Journal of Economics, 28, 472-88.

Lambertini, L. (2003), The Monopolist's Optimal R\&D Portfolio, Oxford Economic Papers, 55, 561-78.

MacDonald, G.M. and A. Slivinsky (1987), The Simple Analytics of Competitive Equilibrium with Multiproduct Firms, American Economic Review, 77, 941-53.

Majerus, D. (1988), Price vs Quantity Competition in Oligopoly Supergames, Economics Letters, 27, 293-97.

Maskin, E. and J. Riley (1984), Monopoly with Incomplete Information, RAND Journal of Economics, 15, 171-96.

Mussa, M. and S. Rosen (1978), Monopoly and Product Quality, Journal of Economic Theory, $18,301-17$.

Okuguchi, K. and F. Szidarovsky (1990), The Theory of Oligopoly with Multiproduct Firms, Lecture Notes in Economics and Mathematical Systems vol. 342, Heidelberg, SpringerVerlag.

Panzar, J. (1989), Technological Determinants of Firm and Industry Structure, in R. Schmalensee and R. Willig (eds.), Handbook of Industrial Organization, vol. 1, Amsterdam, North-Holland, 3-59.

Schumpeter, J.A. (1942), Capitalism, Socialism and Democracy, London, Allen \& Unwin. 
LAMBERTINI Optimal Product Proliferation in Monopoly: A Dynamic Analysis

Singh, N. and X. Vives (1984), Price and Quantity Competition in a Differentiated Duopoly, RAND Journal of Economics, 15, 546-54.

Spence, A.M. (1976), Product Differentiation and Welfare, American Economic Review, 66, 407-14.

Wernerfelt, B. (1986), Product Line Rivalry: A Note, American Economic Review, 76, 842-4. 